\title{
Errata
}

There were errors in the announcement of the UEDA Heart Awards for the Year 2020 in the November 2020 issue. We would therefore like to officially announce that the following 6 articles have been selected for the UEDA Heart Awards for 2020.

\section{Ueda Heart Awards for 2020}

\section{FIRST PLACE}

Activation of DNA Damage Response and Cellular Senescence in Cardiac Fibroblasts Limit Cardiac Fibrosis After Myocardial Infarction

Masato Shibamoto, Tomoaki Higo, Atsuhiko T. Naito, Akito Nakagawa, Tomokazu Sumida, Katsuki

Okada, Taku Sakai, Yuki Kuramoto, Toshihiro Yamaguchi, Masamichi Ito, Yuki Masumura,

Shuichirou Higo, Jong-Kook Lee, Shungo Hikoso, Issei Komuro, and Yasushi Sakata

Int Heart J 2019; 60(4): 944-957.

\section{SECOND PLACE}

Pressure Overload Impairs Cardiac Function in Long-chain Fatty Acid Transporter CD36Knockout Mice

Kazuhiro Nakatani, Daisaku Masuda, Takuya Kobayashi, Masami Sairyo, Yinghong Zhu, Takeshi Okada, Atsuhiko T. Naito, Tohru Ohama, Masahiro Koseki, Toru Oka, Hiroshi Akazawa, Makoto

Nishida, Issei Komuro, Yasushi Sakata, and Shizuya Yamashita

Int Heart J 2019; 60(1): 159-167.

THIRD PLACE (4 winners)

Comparison of Clinical Outcomes Between the Ostial Versus Non-Ostial Culprit in Proximal Left Anterior Descending Artery Acute Myocardial Infarction

Kei Yamamoto, Kenichi Sakakura, Naoyuki Akashi, Yusuke Watanabe, Masamitsu Noguchi, Yousuke

Taniguchi, Hiroshi Wada, Shin-ichi Momomura, and Hideo Fujita

Int Heart J 2019; 60(1): 37-44.

Coronary Artery Calcium Is Associated with Left Ventricular Diastolic Function Independent of Myocardial Ischemia: Assessment by Myocardial Perfusion Single-Photon Emission Computed Tomography

Kazuhiro Nitta, Satoshi Kurisu, Yumiko Nakamoto, Yoji Sumimoto, Atsuhiro Senoo, Hiroki Ikenaga, Fuminari Tatsugami, Ken Ishibashi, Toshiro Kitagawa, Yukihiro Fukuda, Hideya Yamamoto, Kazuo Awai, and Yasuki Kihara

Int Heart J 2019; 60(3): 554-559.

Detection of Pulmonary Hypertension with Systolic Pressure Estimated by Doppler Echocardiography: Comparison with Invasive Mean Pulmonary Artery Pressure Naoko Sawada, Takayuki Kawata, Masao Daimon, Tomoko Nakao, Masaru Hatano, Hisataka Maki, Koichi Kimura, Megumi Hirokawa, Jumpei Ishiwata, Boqing Xu, Yutaka Yatomi, and Issei Komuro Int Heart J 2019; 60(4): 836-844.

Effect of Body Weight Change on Blood Pressure in a Japanese General Population with a Body Mass Index $\geq 22 \mathrm{~kg} / \mathrm{m}^{2}$ : A Community-Based Cohort Study Hidetaka Itoh, Hidehiro Kaneko, Hiroyuki Kiriyama, Koki Nakanishi, Yoshiko Mizuno, Masao Daimon, Hiroyuki Morita, Nobutake Yamamichi, and Issei Komuro

Int Heart J 2019; 60(1): 37-44. 